CERN-TH/96-284

UMN-TH-1517/96

TPI-MINN-96/19

hep-ph/9610410

\title{
ACCELERATOR CONSTRAINTS ON NEUTRALINO DARK MATTER*
}

\author{
J. Ellis \\ TH Division, CERN, CH-1211 Geneva 23 \\ T. Falk and K.A. Olive \\ School of Physics and Astronomy, University of Minneapolis, \\ Minneapolis, MN 55455, USA \\ M. Schmitt \\ PPE Division, CERN, CH-1211 Geneva 23
}

\begin{abstract}
The constraints on neutralino dark matter $\chi$ obtained from accelerator searches at LEP, the Fermilab Tevatron and elsewhere are reviewed, with particular emphasis on results from LEP 1.5. These imply within the context of the minimal supersymmetric extension of the Standard Model that $m_{\chi} \geq 21.4$ $\mathrm{GeV}$ if universality is assumed, and yield for large $\tan \beta$ a significantly stronger bound than is obtained indirectly from Tevatron limits on the gluino mass. We update this analysis with preliminary results from the first LEP $2 \mathrm{~W}$ run, and also preview the prospects for future sparticle searches at the LHC.
\end{abstract}

* Presented by J.E. at the Workshop on the Identification of the Dark Matter, Sheffield, September 1996.

CERN-TH/96-284

UMN-TH-1517/96

TPI-MINN-96/19

October 1996 


\title{
ACCELERATOR CONSTRAINTS ON NEUTRALINO DARK MATTER $^{a}$
}

\author{
J. Ellis \\ TH Division, CERN, CH-1211 Geneva 23, Switzerland
}

T. Falk

School of Physics and Astronomy, University of Minneapolis, Minneapolis, MN $55455, U S A$

K.A. Olive

School of Physics and Astronomy, University of Minneapolis, Minneapolis, MN $55455, U S A$

M. Schmitt

PPE Division, CERN, CH-1211 Geneva 23, Switzerland

\begin{abstract}
The constraints on neutralino dark matter $\chi$ obtained from accelerator searches at LEP, the Fermilab Tevatron and elsewhere are reviewed, with particular emphasis on results from LEP 1.5. These imply within the context of the minimal supersymmetric extension of the Standard Model that $m_{\chi} \geq 21.4 \mathrm{GeV}$ if universality is assumed, and yield for large $\tan \beta$ a significantly stronger bound than is obtained indirectly from Tevatron limits on the gluino mass. We update this analysis with preliminary results from the first LEP $2 \mathrm{~W}$ run, and also preview the prospects for future sparticle searches at the LHC.
\end{abstract}

\section{Theoretical Framework}

We work within the context of the minimal supersymmetric extension of the Standard Model (MSSM) 1 , whose gauge interactions are the same as those in the Standard Model, and whose Yukawa interactions are derived from a superpotential that conserves $R$ parity and includes a term that mixes the two Higgs superfields: $\mu H_{1} H_{2}$. We presume that the lightest supersymmetric particle (LSP) is a neutralino $\chi$, namely, the lightest of the mixtures $\chi_{i}: i=1, \ldots, 4$ of the $U(1)$ gaugino $\tilde{B}$, the neutral $S U(2)$ gaugino $\tilde{W}_{3}$, and the two neutral Higgsinos $\tilde{H}_{1,2}$, found by diagonalizing the mass matrix

${ }^{a}$ Presented by J.E. at the Workshop on the Identification of the Dark Matter, Sheffield, September 1996. 


$$
\left(\begin{array}{cccc}
M_{2} & 0 & \frac{-g_{2} v_{2}}{\sqrt{2}} & \frac{g_{2} v_{1}}{\sqrt{2}} \\
0 & M_{1} & \frac{g^{\prime} v_{2}}{\sqrt{2}} & \frac{-g^{\prime} v_{1}}{\sqrt{2}} \\
\frac{-g_{2} v_{2}}{\sqrt{2}} & \frac{g^{\prime} v_{2}}{\sqrt{2}} & 0 & \mu \\
\frac{g_{2} v_{1}}{\sqrt{2}} & \frac{-g^{\prime} v_{1}}{\sqrt{2}} & \mu & 0
\end{array}\right)
$$

where $g_{2}, g^{\prime}$ are the $S U(2)$ and $U(1)$ gauge couplings, $v_{1,2}=<0\left|H_{1,2}\right| 0>$ are the Higgs vacuum expectation values whose ratio we denote by $\tan \beta=v_{2} / v_{1}$, and $M_{1,2}$ are the soft supersymmetry-breaking $U(1)$ and $S U(2)$ gaugino masses. The mass matrix for the charginos $\chi^{ \pm}$, which are mixtures of the charged yinos $\tilde{W}^{ \pm}$and Higgsinos $\tilde{H}^{ \pm}$are also characterized by $g_{2}, v_{1,2}$ and $M_{2}$. We make here the conventional universality assumption that $M_{1}=M_{2} \equiv m_{1 / 2}$ at the supersymmetric GUT scale, so that their physical values are renormalized 1 :

$$
M_{2}: M_{1}: m_{1 / 2}=\alpha_{2}: \alpha_{1}: \alpha_{G U T}
$$

We also assume universality for the soft supersymmetry-breaking scalar squared masses: $m_{0_{i}}^{2} \equiv m_{0}^{2}$ at the sppersymmetric GUT scale, so that the physical values are renormalized 1 :

$$
m_{0_{i}}^{2} \simeq m_{0}^{2}+C_{i} m_{1 / 2}^{2}+\mathrm{D} \text { terms }
$$

Theoretically, this assumption is more questionable than (2), apd possible implications of its relaxation are discussed here by Bottino $\mathrm{B}$.

\section{Experimental Lower Bound from LEP}

An important experimental step forward in constraining neutralinos was made possible by the LEP 1.5 run in late 1995 4 . Previously, searches for $Z^{0} \rightarrow \chi^{+} \chi^{-}$and $\chi \chi^{\prime}$ at LEP 1 had not been able to establish a model-independent lower bound on $m_{\chi}$, as seen in Fig. 15 . Nor, indeed, were the LEP 1.5 searches for $e^{+} e^{-} \rightarrow \chi^{+} \chi^{-}$and $\chi_{i} \chi_{j}$ (whose cross section depends on the sneutrino mass $m_{\tilde{\nu}}$ ) able alone to establish a lower bound, as also shown in Fig. 15. However, the LEP 1.5 data did serve to fill in a 'wedge' of parameter space left uncovered by LEP 1 data for $\mu<0, \tan \beta \leqslant 2$, as seen in Fig. 2. This was sufficient for the ALEPH collaboration 5 to quote a lower limit

$$
m_{\chi} \geq 12.8 \mathrm{GeV}
$$

for $m_{\tilde{\nu}}=200 \mathrm{GeV} \Phi$. In fact, as discussed in l and seen in Fig. 3, there was still a small loophole for $1<\tan \beta<1.02$ which could not be excluded

\footnotetext{
${ }^{b}$ This analysis also excluded the theoretically-interesting possibility that $\mu=0$.
} 


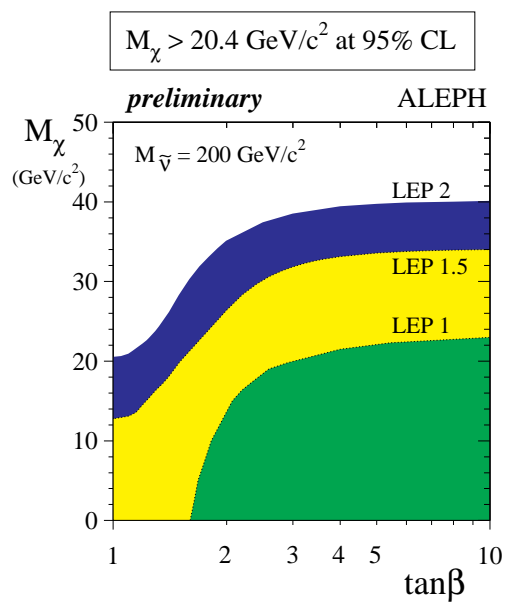

Figure 1: Experimental lower bound 0 on the neutralino mass: note that neither LEP 1 nor LEP 1.5 data by themselves impose a non-zero lower bound, though there combination does, modulo the loopholes discussed in the text.

by the ALEPH LEP 1.5 data alone, though it could be excluded by combining them with data from the other LEP collaborations, or by other considerations 6 . Of greater concern was a larger loophole thet appeared when $m_{0} \sim 60 \mathrm{GeV}$ and $\tan \beta \sim \sqrt{2}$, as seen in Fig. 4 目, which was due to a loss of sensitivity to $\chi^{ \pm}$production becaupg of the invisibility of $\chi^{ \pm} \rightarrow \tilde{\nu}+$ soft $e^{ \pm}$decays made manifest in Fig. 55 .

\section{Phenomenological Analysis}

We 6 have attempted to eliminate these two loopholes and strengthen the ALEPH lower bound on $m_{\chi}$ by supplementing the Aleph analysis 5 with additional phenomenological inputs. For example, the neutrino counting analysis at the $Z^{0}$ peak not only constrains $Z^{0} \rightarrow \chi \chi$ decay, but also $Z^{0} \rightarrow \tilde{\nu} \overline{\tilde{\nu}}$ decay. Taking $N_{\nu}=2.991 \pm 0.016$ U, we found that $m_{\tilde{\nu}}>43.1 \mathrm{GeV}$, if three degenerate flavours of neutrinos are assumed, as expected in the MSSM with universality Also, LEP 1.5 established new lower limits on charged slepton masses 1 . As seen in Fig. 6 for the case $\tan \beta=\sqrt{2}$, these two constraints between them limit the loophole allowing $m_{\chi}=0$ when $m_{\chi^{ \pm}}>m_{\tilde{\nu}}$, but do not exclude it. However, this possibility is excluded by searches at lower centre-of-mass energies for $e^{+} e^{-} \rightarrow \gamma+$ nothing by the AMY and other experiments 8 , which can be interpepted as upper limits on $\chi \chi$ production mediated by selectron exchange 9 . These exclude a zone in the $\left(m_{1 / 2}, m_{0}\right)$ plane which finally 


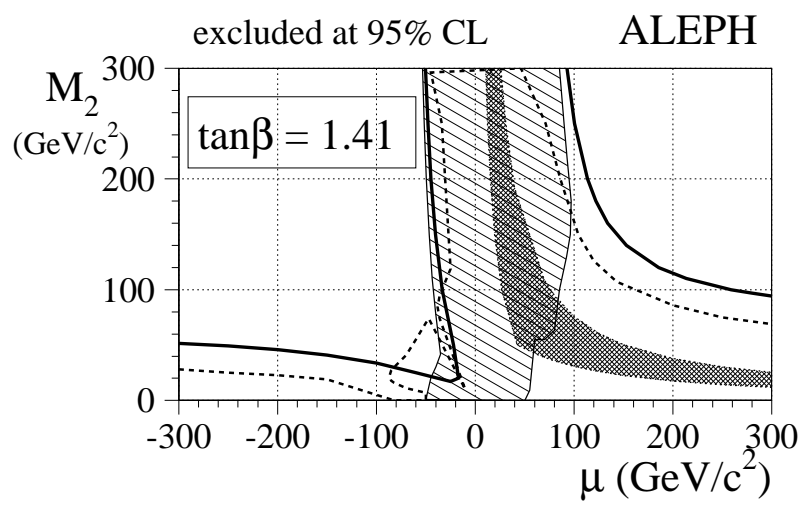

Figure 2: The region of the $\left(\mu, M_{2}\right)$ plane excluded by 5 on the basis of searches for charginos and neutralinos at LEP1 and LEP 1.5.

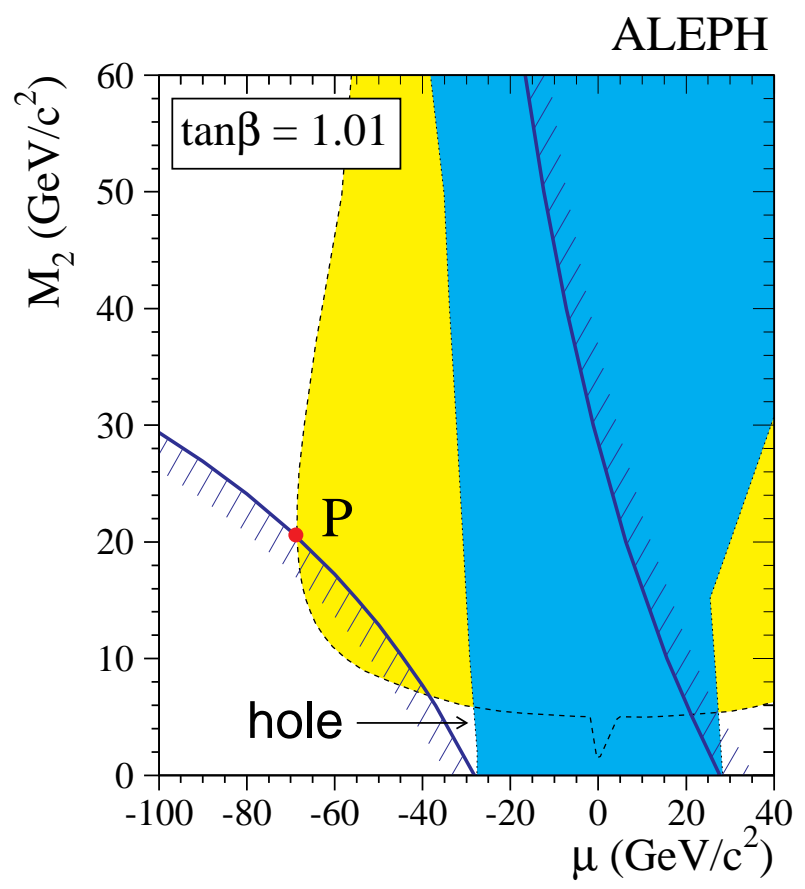

Figure 3: The small loophole near $m_{1 / 2}=0$ for $1<\tan \beta<1.02$ in the LEP 1.5 analysis by ALEPHE. 


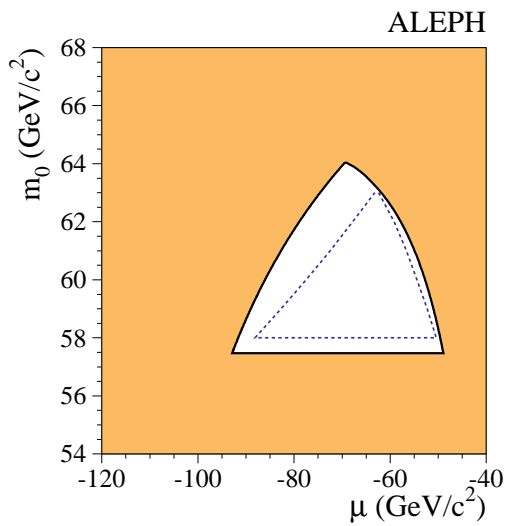

Figure 4: The larger loophole for $\tan \beta \sim \sqrt{2}$ and $m_{0} \sim 60 \mathrm{GeV}$ where the ALEPH analysis 5 allows $m_{1 / 2}=0$.

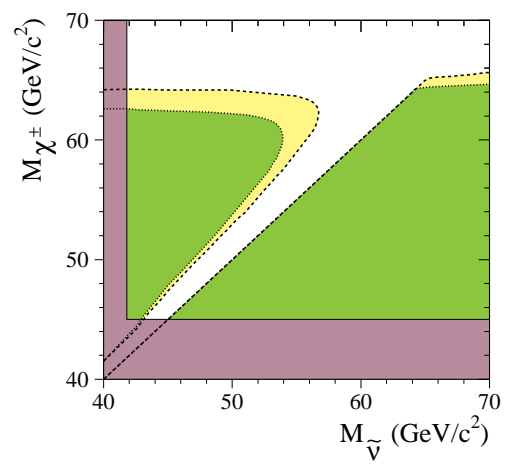

Figure 5: The loss of sensitivity in the ALEPH $\chi^{ \pm} \rightarrow \tilde{\nu}+\operatorname{soft} e^{ \pm}$search 5, which is responsible for the loophole shown in Fig. 4. 


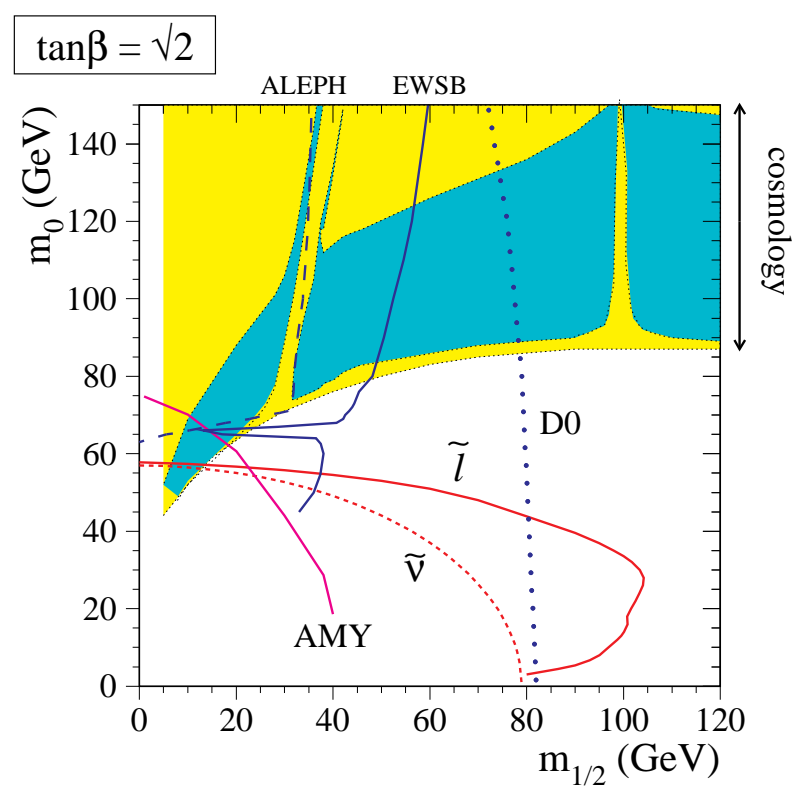

Figure 6: The domain of the $\left(m_{1 / 2}, m_{0}\right)$ plape for $\mu<0$ and $\tan \beta=\sqrt{2}$ that is excluded by ALEPH chargino and neutralino searches 1 (long-dashed line), by the $Z^{0}$ limit on $m_{\tilde{\nu}}$ (short-dashed line), by the LEP limits 4 on slepton production (solid lime), by single-photon measurements 8 (grey line), and by the D0 limit on the gluino mass 10 (dotted line). the region of the plane in which $0.1<\Omega_{\chi} h^{2}<0.3$ for some experimentally-allowed value of $\mu<0$ is light-shaded, whilst the dark-shaded region if for $\mu$ determined by dynamical EWSB. the constraint derived from the ALEPH searches 1 when dynamical EWSB is imposed is also shown as a solid line 6 .

eliminates the possibility that $m_{\chi}=0$, as demonstrated in Fig. 6 6

To go further, one must take account other phenomemological constraints. Since we are interested in neutralino dark matterl, it is natural to impose with first priority the requirement that the relic cosmological density $\rho_{\chi}$ lie in a range of interest to astrophysicists. We base our analysis on theories of structure formation based on inflation, with total mass density $\Omega \simeq 1$. Models with mixed hot and cold dark matter and a flat spectrum of primordial perturbations, with a cosmological constant and cold dark matter, and with cold dark matter and a tilted perturbation spectrum, all favour the range 11

$$
0.1 \leq \Omega_{\chi} h^{2} \leq 0.3
$$

which we select for our analysis. 


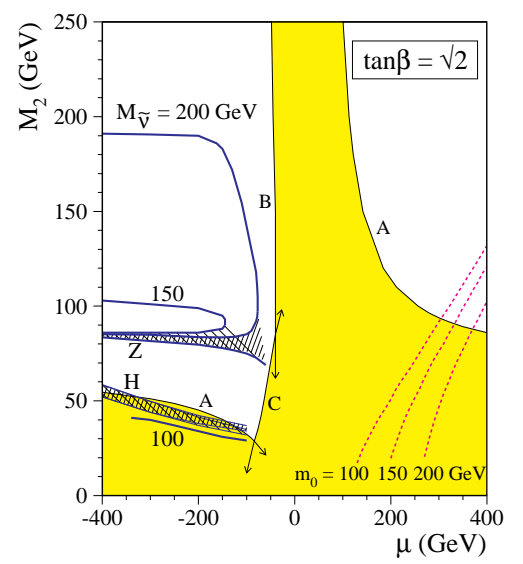

Figure 7: The region of the $\left(\mu, M_{2}\right)$ plane excluded by direct searches 4 for $(\mathrm{A})$ charginos at LEP 1.5 , (B) neutralinos at LEP 1.5 and (C) $Z^{0}$ decays into $\chi \chi^{\prime}$ at LEP 1 for $\tan \beta=\sqrt{2}$ are indicated by thin solid lines. Contours of $m_{\tilde{\nu}}$ (in GeV) required in the MSSM to obtain $\Omega_{\chi} h^{2}=0.2$ for $\mu<0$ are indicated by thick solid lines. The hatched regions indicate where the $Z^{0}$ and Higgs poles suppress the relic density. Values of $\mu$ required by dynamical EWSB for the indicated values of $m_{0}$ (in $\mathrm{GeV}$ ) are shown as short-daghed lines for $\mu<0$ : identical values would be required for $\mu<0$.

The relic $\chi$ density is controlled annihilations via $\tilde{q}, \tilde{\ell}, Z^{0}$, neutralino, chargino and Higgs exchanges 12 . Their general trend is to favour some range of $m_{0}$ which depends 0 on $\mu$ and $m_{1 / 2}$ for any given value of $\tan \beta$ 13, as illustrated in Fig. 7 . Note, however, that this trend is punctuated by holes due to annihilation via direct-channel $Z^{0}$ and Higgs poles, which are most important when $m_{\chi} \sim M_{Z, H} / 2$. Over a wide range of $m_{\chi}$, these cannot be neglected, and require a careful treatment that goes beyond a simple power-series expansion in the $\chi$ momenta 14 . Fig. 6 displays as the light-shaded region the constraint imposed by the cosme logical density requirement (5) in the $\left(m_{1 / 2}, m_{0}\right)$ plane for $\tan \beta=\sqrt{2}$. We see that it tends to keep $m_{0}$ away from the dangerous region where $m_{\tilde{\nu}} \lesssim m_{\chi^{ \pm}}$, without eliminating it entirely.

So far, we have not introduced any further theoretical assumptions into the MSSM, beyond those of universality for the scalar and gaugino masses. It is attractive to hypothesize that electroweak symmetry breaking (EWSB) is driven dynamically by the renormalization-group running of the soft supersymmetry-breaking mass of the Higgs boson coupled to the top quark 15 . This EWSB assumption may be regarded effectively as fixing $\mu$ for given values of the other MSSM parameters 16 , as illustrated on the right-hand side of Fig. 7 for $\tan \beta=\sqrt{2}$, tending to bound it 


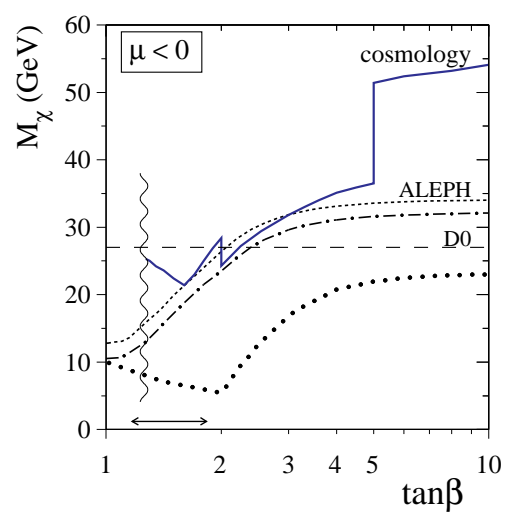

Figure 8: The ALEPH lower limit on $m_{\chi} 5$ for $\mu<0$ and for large $m_{\tilde{\nu}}$ (short-dashed line) is compared, as a function of $\tan \beta$, with the results obtained in the text by making different phenomenofogical and theoretical inputs. The dotted line is obtained by combining the AMY constraint 8 with other unsuccessful searches for sleptons and sneutrinos: it excludes the region of $\tan \beta$, indicated by a double arrow, where the ALEPH experimental limit does not exclude $m_{\chi}=0$. The dash-dotted line is obtained by rquiring also that the cosmological relic neutralino density fall within the preferred range 11 . The solid line it obtained by combining these experimental and cosmological inputs with the assumption 15 of dynamical electroweak symmetry breaking. The vertical wavy line indicates the lower limit on $\tan \beta$ in such dynamical electroweak symmetry breakingmodels. The horizontal long-dashed line is that obtained from the D0 gluino search 10 , assuming gaugino mass universality.

away from the dangerous regions. In particular, note that the EWSB assumption cannot be implemented for any value of $\mu$ when $\tan \beta \lesssim 1.2$ for $m_{t} \geq 161 \mathrm{GeV}$ as indicated by experiment, which excludes the small loophole for $\tan \beta \leq 1.02$ mentioned earlier 6 . The EWSB assumption may be implemented either in isolation or in combination with the cosmological constraint (5), as seen in Fig. 6. Taken in isolation, EWSB also reduces the extent of the loophole where $m_{\tilde{\nu}} \lesssim m_{\chi^{ \pm}}$, without eliminating it completely. However, cosmology (5) in combination with EWSB is considerably more stringent. The channels through the darker-shaded region in Fig. 76 reflect the positions of the direct-channel Higgs and $Z$ poles, whose locations are strongly constrained in this case. Because of the immobility of these channels, the upper limit in (5) on the cosmological density provides an upper limit on $m_{0}$ for generic values of $m_{1 / 2}$, which was not the case before the imposition of EWSB.

As an example of the application of the above constraints, let us consider the specific case $\tan \beta=\sqrt{2}$, for which EEP 1 alone allowed $m_{\chi}=0$. The ALEPH analysis for $m_{\tilde{\nu}}=200 \mathrm{GeV} 5$, which is not a conservative assumption, as can be seen from the figures, yielded $m_{\chi} \gtrsim 17$ 
$\mathrm{GeV}$. If we relax this assumption so as to allow any value of $m_{\tilde{\nu}}$, but implement all the other experimental constraints especially that from $e^{+} e^{-} \rightarrow \gamma+$ nothing, we find $m_{\chi} \gtrsim 5 \mathrm{GeV}$. This lower bound can be strengthened by requiring the cosmological constraint (5), which yields $m_{\chi} \gtrsim 16 \mathrm{GeV}$, modulo a small fraction of the previous experimental loophole. Finally, if we combine cosmology with the assumption of dynamical EWSB, we find $m_{\chi} \gtrsim 24 \mathrm{GeV}$.

Our conclusions for general $\tan \beta$ are summarized in Fig. 8. We find that the limit $m_{1 / 2} \rightarrow$ Ois excluded, as well as the limit $\mu \rightarrow 0$. We find an absolute lower limit

$$
m_{\chi} \geq 21.4 \mathrm{GeV}
$$

which is attained for $\tan \beta \simeq 1.6$. We see in Fig. 8 that, for generic values of $\tan \beta$, this LEP bound is stronger than what could be inferred, assuming gaugino mass universality, from the unsuccessful D0 search for gluinos $\tilde{g}$. This improvement is particularly marked for large values of $\tan \beta$, and is also significant for small $\tan \beta$, particularly if LEP cop straints on supersymmetric Higgs boson masses are taken into account at the price of additional sensitivity to theoretical assumptions.

The conclusion (d) has potentially-important implications for the design of direct experimental searches for supersymmetric dark atter. It diminishes the priority of a sensitivity to low $m_{\chi} \lesssim 10 \mathrm{GeV} 18$, and it indicates that higher nucleon recoil energies may have a higher a priori probability. Taken together, these observations indicate that one might be prepared to sacrifice a lower threshold recoil energy on the altar of a larger detector mass.

\section{Update Including Preliminary LEP 2W Results}

During the summer of 1996, LEP was run for the first time at an energy above the $W^{+} W^{-}$threshold: $E_{c m}=161 \mathrm{GeV}$, which we term LEP $2 \mathrm{~W}$. The first results of searches during this fun for supersymmetric particles were presented at the Warsaw ICHEP 19 and Minneapolis DPF 20 conferences A, and preliminary summaries of their analyses have now been presented at CERN by all the LEP collaborations 21. These have included new upper limits on chargino, neutralino and slepton production, implying for example a new lower limit

$$
m_{\chi^{ \pm}} \gtrsim 80 \times f\left(\mu, m_{\tilde{\nu}}, \tan \beta\right) \mathrm{GeV}
$$

This and the new preliminary upper limits on $\sigma\left(e^{+} e^{-} \rightarrow \chi_{i} \chi_{j}\right)$ can be used to establish a new preliminary exclusion domain in the $\left(\mu, m_{1 / 2}\right)$

\footnotetext{
${ }^{c}$ Et was commented in the first paper in 20 that general features of the ALEPH 1.5 analysis 5 were insensitive to moderate violations of universality between $M_{1,2}$.
} 


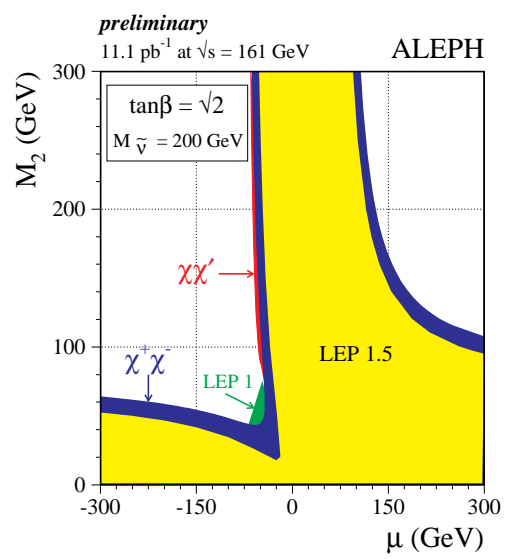

Figure 9: The region of the $\left(\mu, M_{2}\right)$ plane excluded by the prelininary analysis of searches for charginos and neutralinos at LEP $2 \mathrm{~W} 21$.

plane 21, as shown in Fig. 9. This enables the previous purely experimental lower limit (4) to be strengthened to

$$
m_{\chi} \gtrsim 20 \mathrm{GeV}
$$

for the case $m_{\tilde{\nu}}=200 \mathrm{GeV}$, as shown in Fig. 1 .

Furthermore, this limit does not vary much for $m_{\tilde{\nu}} \gtrsim 80 \mathrm{GeV}$. Moreover, the two loopholes where $M_{2}=0$ was formerly possible, for $1<\tan \beta<1.02$ at large $m_{\tilde{\nu}}$ and for $\tan \beta \sim \sqrt{2}$ and $\sim 60 \mathrm{GeV}$, are now both closed by preliminary LEP $2 \mathrm{~W}$ data alone 21 , without the need to combine them with data from other experiments or to use supplementary theoretical assumptions.

We have embarked on an improved phenomenological analysis22, with the aim of seeing how much the bound (\$) may be strengthened by combining the full set of 1996 LEP 2 data with the cosmological and dynamical EWSB assumptions invoked earlier. Fig. 10 displays a rough assessment of the impact of the preliminary LEP $2 \mathrm{~W}$ data on our previous analysis of the excluded domains in the $\left(m_{0}, m_{1 / 2}\right)$ plane shown in Fig. 6. We see that the chargino and neutralino limits do not by themselves improve significantly the previous absolute lower limit on $m_{1 / 2}$, even if our cosmolegical. assumption (5) is invoked. However, the LEP $2 \mathrm{~W}$ slepton limits 21 do represent significant new constraints. We have not yet implemented dynamical EWSB in this updated analysis, in which we plan-to include constraints from searches fof supersymmetric Higgs bosons 21 , which may be significant at low $\tan \beta$. 


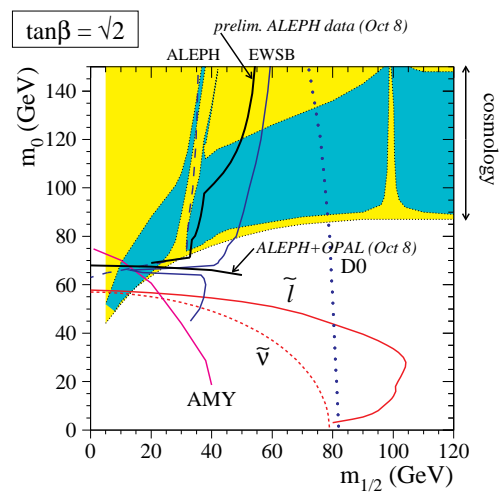

Figure 10: Update of Fig. 6, including rough estimates of the potential impact of the LEP $2 \mathrm{~W}$ searches for charginos, neutratipos and sleptons. These personal assessments of preliminary data 21 , which will be refined 22 as and when these data are published.

\section{Prospects for LHC Searches}

The LHC is designed to have a centre-of-mass energy of $14 \mathrm{TeV}$ for $p p$ collisions, at a luminosity $\mathcal{L} \simeq 10^{34} \mathrm{~cm}^{-2} \mathrm{~s}^{-1}$, enabling it to explore physics at energy scales $\lesssim 1 \mathrm{TeV}$. In particular, detailed calculatipms of the cross sections for the production of supersymmetric particles 23 are available, and it seems that the LHC should be able to detect the pir production of squarks $\tilde{q}$ and gluinos $\tilde{g}$ if their masses are $\lesssim 2 \mathrm{TeV} 24$. Using the proportionality between $m_{\tilde{g}}$ and $m_{\chi}$ expected on the basis of gaugino mass universality, this sensitivity corresponds to a physics reach up to

$$
m_{\chi} \simeq 300 \mathrm{GeV}
$$

thereby covering most of the range of interest for supersymmetric dark matter experiments.

The primary sparticle signature studied up now has been the classic missing-energy signature of LSP emission 24, which is expected to stand out well above the Standard Model and detector backgrounds, as seen in Fig. 11. Recent studies indicate that this may used to give quite an accurate estimate of the lighter of $m_{\tilde{q}}$ and $m_{\tilde{g}} 25$. The potential importance and interest of cascade sparticle decays via intermediate states have been apparent for some time 26 , and their signatures, such as $\ell^{ \pm} \ell^{ \pm}, 3 \ell$ and $Z^{0}+E_{T_{m i s s}}$ final states, are now-peing studied in greater detail by the ATLAS and CMS collaborations 27.

For particular values of the MSSM parameters, cascade decays may enable the masses of several supersymmetric particles to be determined 


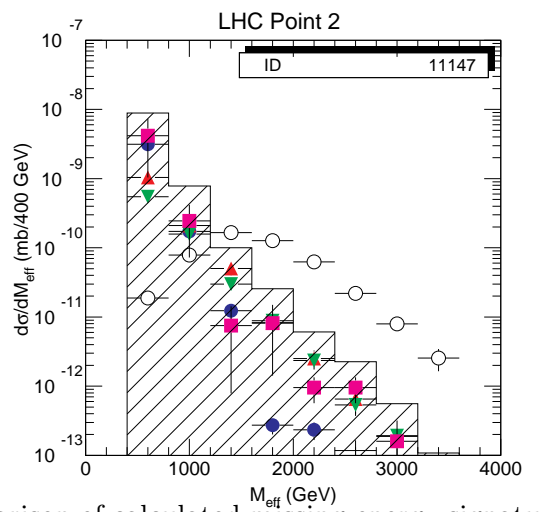

Figure 11: Comparison of calculated $\stackrel{\mathrm{M}_{\text {eff }}(\mathrm{GeV})}{\text { missing-energy }}$ irgnature due to $\tilde{q}, \tilde{g}$ production at the LHC with Standard Model and detector backgrounds 25, in a model with dynamical EWSB and $m_{0}=400 \mathrm{GeV}, m_{1 / 2}=400 \mathrm{GeV}, \tan \beta=10$ and $\mu>0$.

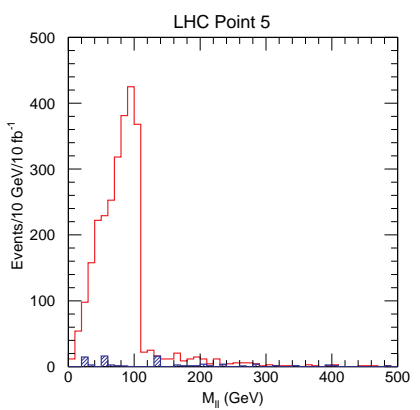

Figure 12: Spectrum of $\ell^{+} \ell^{-}$expected to be produced in $\chi_{2} \rightarrow \chi$ decays at the LHC 25 , in a model with dynamical EWSB and $m_{0}=100 \mathrm{GeV}, m_{1 / 2}=300 \mathrm{GeV}, \tan \beta=2.1$ and $\mu<0$.

simultaneously with high precision 22. One generic possibility is that the cascade includes $\chi_{2} \rightarrow \chi^{+} \ell^{+} \ell^{-}$decays, which have a sharp end point in $m_{\ell \ell}$, as seen in Fig. 1225. This may be used to measure $m_{\chi_{2}}-m_{\chi}$ with a systematic uncertainty $\lesssim 50 \mathrm{MeV}$ ! It may then be possible to measure accurately other sparticle masses by reconstructing the rest of the decay chain, for example the $\tilde{b}$ and $\tilde{g}$ masses in $\tilde{g} \rightarrow \tilde{b}+\chi_{2}$ decay 28 . In this way, the LHC may be able to measure several combinations of MSSM parameters with high precision, enabling the relic $\chi$ density to be calculated more accurately, providing the ultimate accelerator constraints on neutralino dark matter. 
1. For reviews, see: H.P. Nilles, Physics Reports 110 (1984) 1; E.H. Haber and G.L. Kane, Physics Reports 117 (1985) 75.

2. J. Ellis, J.S. Hagelin, D.V. Nanopoulos, K.A. Olive and M. Srednicki, Nucl.Phys. B238 (1984) 453.

3. A. Bottino, talk at this meeting; V. Berezinskii et al., Ann.Phys. 5 (1996) 1.

4. ALEPH Collaboration, D. Buskulic et al., Phys.Lett. B373 (1996) 246 ;

OPAL Collaboration, G. Alexander et al., Phys.Lett. B377 (1996) 181 ;

L3 Collaboration, M. Acciari et al., Phys.Lett. B377 (1996) 289; DELPHI Collaboration, P. Abreu et al., CERN Preprint PPE/9675 (1996).

5. ALEPH Collaboration, D. Buskulic et al., CERN Preprint PPE/9683 (to appear in Zeitschrift für Physik).

6. J. Ellis, T. Falk, K.A. Olive and M. Schmitt, CERN Preprint TH/96-102 (to appear in Phys.Lett. ).

7. The LEP Collaborations ALEPH, DELPHI, L3, OPAL and the LEP Electroweak Working Group, CERN Preprint PPE/95-172 (1995).

8. AMY Collaboration, Y. Sugimoto et al., Phys.Lett. B369 (1996) 86.

9. P. Fayet, Phys.Lett. B69 (1977) 489;

J. Ellis and J.S. Hagelin, Phys.Lett. B122 (1983) 303;

K. Grassie and P.N. Pandita, Phys.Rev. D30 (1984) 22.

10. D0 Collaboration, S. Abachi et al., contributed paper No. 434 to the Int. Europhys. Conf. on High Energy Physics (Brussels, 1995).

11. For a review and references, see V. Berezinskii et al., CERN Preprint TH/96-42, hep-ph/9603342.

12. J. McDonald, K.A. Olive and M. Srednicki, Phys.Lett. $\mathbf{B 2 8 3}$ (1992) 80.

13. See, for example, J. Ellis and L. Roszkowski, Phys.Lett. $\mathbf{B 2 8 3}$ (1992) 252.

14. K. Griest and D. Seckel, Phys.Rev. D43 (1991) 3191;

P. Goldolo and G. Gelmini, Nucl.Phys. B360 (1991) 145.

15. L.E. Ibañez and G.G. Ross, Phys.Lett. B110 (1982) 215;

L.E. Ibañez, Phys.Lett. B118 (1982) 73;

J. Ellis, D.V. Nanopoulos and K. Tamvakis, Phys.Lett. B121 (1983) 123;

L. Alvarez-Gaumé, J. Polchinski and M.B. Wise, Nucl.Phys. B221 (1983) 495;

K. Inoue, A. Kakuto, H. Komatsu and S. Takeshita, Progr.Theor.Phys. 68 (1982) 927.

16. For a review and references, see: J. Ellis, Rapporteur Talk at 
the International Symposium on Lepton and Photon Interactions at High Energies, Beijing 1995, CERN Preprint TH/95-316, hep$\mathrm{ph} / 9512335$.

17. See, for example, L. Roszkowski, Phys.Lett. 252 (1990) 471. and talk at this meeting.

18. C. Absmeier et al., Max-Planck-Institut, München Preprint MPIPHE-95-15 (1995);

see also A. Gabutti et al., hep-ph/9602432.

19. W.-D. Schlatter, M. Green, Contributions to appear in the Proceedings of the International Conference on High Energy Physics, (Warsaw, 1996).

20. J. Nachtman, W. Orejudos, Contributions to appear in the Proceedings of the Annual Meeting of the Division of Particles and Fields of the American Physical Society, (Minneapolis, 1996).

21. ALEPH, DELPHI, L3 and OPAL collaborations, presentations at open session of the CERN LEP Experiments Committee, Oct. 8th, 1996

See, for example, OPAL collaboration, K. Ackerstaff et al., CERN Preprint PPE/96-135.

22. J. Ellis, T. Falk, K.A. Olive and M. Schmitt, in preparation.

23. W. Beenakker, R. Höpker, M. Spira and P. Zerwas, Phys.Rev.Lett. 74 (1995) 2905 and hep-ph/9505416.

24. CMS Collaboration, Technical Proposal, LHCC/P1 (1994); ATLAS collaboration, Technical Proposal, LHCC/P2 (1994).

25. F.E. Paige, hep-ph/9609373, to appear in the Proceedings of the Workshop on Future Directions in High Energy Physics (Snowmass, 1996).

26. H. Baer, C.-H. Chen, F. Paige and X. Tata, Phys.Rev. D52 (1995) 2746 and D53 (1996) 6241.

27. Open session of the CERN LHC Experiments Committee, Oct. 30th, 1996.

28. I. Hinchliffe, F.E. Paige, M.D. Shapiro, J. Soderqvist and W. Yao, to appear in the Proceedings of the Workshop on Future Directions in High Energy Physics (Snowmass, 1996). 